\title{
Direct Temporal Mode Measurement for the Characterization of Temporally Multiplexed High Dimensional Quantum Entanglement in Continuous Variables
}

\author{
Nan Huo๑, ${ }^{1}$ Yuhong Liu, ${ }^{1}$ Jiamin Li,${ }^{1}$ Liang Cui, ${ }^{1}$ Xin Chen, ${ }^{2}$ \\ Rithwik Palivela, ${ }^{3}$ Tianqi Xie, ${ }^{1}$ Xiaoying Li, ${ }^{1, *}$ and Z. Y. Ou ${ }^{1,2, \dagger}$ \\ ${ }^{1}$ College of Precision Instrument and Opto-Electronics Engineering, Key Laboratory of Opto-Electronics Information Technology, \\ Ministry of Education, Tianjin University, Tianjin 300072, People's Republic of China \\ ${ }^{2}$ Department of Physics, Indiana University-Purdue University Indianapolis, Indianapolis, Indiana 46202, USA \\ ${ }^{3}$ Carmel High School, 520 East Main Street, Carmel, Indiana 46033, USA
}

(Received 24 October 2019; accepted 29 April 2020; published 27 May 2020)

\begin{abstract}
Field-orthogonal temporal mode analysis of optical fields has recently been developed for a new framework of quantum information science. However, so far, the exact profiles of the temporal modes are not known, which makes it difficult to achieve mode selection and demultiplexing. Here, we report a novel method that measures directly the exact form of the temporal modes. This, in turn, enables us to make mode-orthogonal homodyne detection with mode-matched local oscillators. We apply the method to a pulse-pumped, specially engineered fiber parametric amplifier and demonstrate temporally multiplexed multidimensional quantum entanglement of continuous variables in telecom wavelength. The temporal mode characterization technique can be generalized to other pulse-excited systems to find their eigenmodes for multiplexing in the temporal domain.
\end{abstract}

DOI: $10.1103 /$ PhysRevLett.124.213603

Any electromagnetic field, no matter what state (quantum or classical) it is in, is first characterized by its modes, which are a special class of solutions to the Maxwell equation [1]. Modes of the field become especially important when quantum fields are involved because quantum interference requires indistinguishability of photons, whereas modes are distinct features of photons. While spatial modes are easily defined through boundary conditions, as in optical cavity and waveguide systems, temporal modes are less concerned because they are usually dealt with through spectral analysis in the frequency domain.

On the other hand, it was discovered recently that fieldorthogonal temporal modes of electromagnetic fields form a new framework for quantum information [2]. Temporal mode analysis offers a straightforward way with intrinsically decoupled modes [3-5] in describing pulse-pumped parametric processes. Parametric processes, ever since first proposed by Yuen [6], have been by far the most common processes to generate experimentally a variety of quantum states of light [7-13] and are widely used in quantum information and communication [14,15], quantum simulation [16], and quantum metrology for precision measurement [17]. It should be pointed out that temporal mode analysis was performed on a pulse-pumped parametric down-conversion of a femtosecond-frequency comb in an optical cavity with a complicated quantum wavelength multiplexing method $[18,19]$, which indirectly revealed the eigen-temporal mode structure as the supermodes. Temporal mode functions of photons in spontaneous parametric processes were also obtained indirectly by making the singular-value decomposition of the joint spectral function that can be measured directly [20]. However, as we shall see later, this cannot be applied to high gain parametric processes where quantum entanglement is exhibited in the continuous variables. So, temporal modes of a system have never been directly measured so far.

Moreover, temporal modes are not easily separated, even though quantum pulse gates [21-24] through nonlinear interaction processes have recently been invented to distinguish them with some success. The consequence is that the contributions from different temporal modes add, leading to some detrimental effects, such as extra noise due to out-of-sync phases for different modes in optimum quantum noise reduction [5]. On the other hand, homodyne detection can also select out and distinguish the contributions from different temporal modes by a properly matched local oscillator (LO) [5]. However, this requires the knowledge of the exact forms of the temporal modes in order to have a matched LO engineered.

In this Letter, we use a feedback-iteration method with a trial seed pulse to obtain and eventually measure the exact forms of the temporal modes of the two correlated fields generated from a pulse-pumped, single-pass broadband fiber parametric amplifier. We then measure the quantum correlations between the signal and idler fields by performing homodyne detection with LOs engineered to match the specific temporal modes. We observe quantum entanglement in three pairs of mutually orthogonal temporal modes and confirm the independence between different pairs by quantum measurement. 


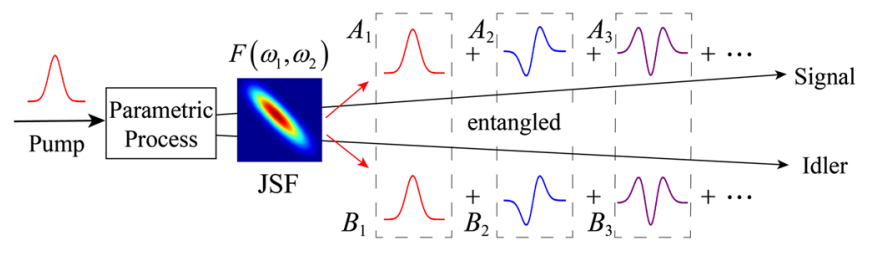

FIG. 1. Conceptual diagram for the entangled states in various temporal modes $\left(A_{k}, B_{k}\right)$ from a pulse-pumped parametric process.

Theoretical background.-Pulse-pumped parametric processes generate two fields dubbed "signal" and "idler", which are quantum mechanically entangled. The spectral profiles of the entangled fields are extremely complicated because of the dispersion-dependent phase matching of the nonlinear medium and the wide spectrum of the pump field. However, this complicated system can always be thought of as superposition of its eigenmodes whose temporal or spectral profiles do not change by the amplifier, as shown in Fig. 1. That is, there exists an independent set of pairwise modes $\left\{\hat{A}_{k}, \hat{B}_{k}\right\}(k=1,2, \ldots)$ for the signal and idler fields that are related by [3-5]

$$
\begin{aligned}
& \hat{A}_{k}^{\text {out }}=\hat{A}_{k}^{\text {in }} \cosh G_{k}+\hat{B}_{k}^{\text {in } \dagger} \sinh G_{k}, \\
& \hat{B}_{k}^{\text {out }}=\hat{B}_{k}^{\text {in }} \cosh G_{k}+\hat{A}_{k}^{\text {in } \dagger} \sinh G_{k},
\end{aligned}
$$

where $\hat{A}_{k} \equiv \int d \omega \psi_{k}(\omega) \hat{a}_{s}(\omega), \hat{B}_{k} \equiv \int d \omega \varphi_{k}(\omega) \hat{a}_{i}(\omega)$ are the annihilation operators for the $k$ th modes of the signal and idler fields with respective eigen-temporal profiles of $f_{k}(\tau) \equiv \int d \omega \psi_{k}(\omega) e^{-i \omega \tau}, g_{k}(\tau) \equiv \int d \omega \varphi_{k}(\omega) e^{-i \omega \tau}$, satisfying the orthonormal relations

$$
\int d \omega \psi_{k}^{*}(\omega) \psi_{k^{\prime}}(\omega)=\delta_{k k^{\prime}}=\int d \omega \varphi_{k}^{*}(\omega) \varphi_{k^{\prime}}(\omega) .
$$

These eigenmodes are exactly the supermodes studied by Roslund et al. [18], which are pairwise entangled and form a multidimensional quantum entangled states.

At relatively low pumping power so that $\left|G_{k}\right| \ll 1$, the eigenfunctions $\left\{\psi_{k}(\omega), \varphi_{k}(\omega)\right\}$ can be obtained by the singular-value decomposition method from the joint spectral function (JSF)

$$
F\left(\omega_{1}, \omega_{2}\right)=G \sum_{k} r_{k} \psi_{k}\left(\omega_{1}\right) \varphi_{k}\left(\omega_{2}\right),
$$

which is defined via the interaction Hamiltonian [3,25]

$$
\frac{1}{i \hbar} \int d t \hat{H}=\int d \omega_{1} d \omega_{2} F\left(\omega_{1}, \omega_{2}\right) \hat{a}_{s}^{\dagger}\left(\omega_{1}\right) \hat{a}_{i}^{\dagger}\left(\omega_{2}\right)+\text { H.c. }
$$

Here, $\left\{r_{k}\right\} \geq 0$ are the mode numbers satisfying the normalization relation $\sum_{k} r_{k}^{2}=1 . G>0$ is a parameter proportional to the peak amplitudes of the pump fields, nonlinear coefficient, and nonlinear medium length. Then, we have $G_{k}=r_{k} G$ with $r_{k}$ independent of $G$.
At high pump power, when stimulated emission dominates, Eq. (1) still holds, but $\left\{r_{k}\right\}$ and $\left\{\psi_{k}(\omega), \varphi_{k}(\omega)\right\}$ now depend on the pump parameter $G[26,27]$.

So far, mode functions $\left\{\psi_{k}\left(\omega_{1}\right), \varphi_{k}\left(\omega_{2}\right)\right\}$ are only obtained in simulations but have never been measured directly. In the following, we will describe a method to directly measure these mode functions experimentally.

Temporal modes determination.-Our procedure to find the mode functions $\psi_{k}(\omega), \varphi_{k}(\omega)$ is based on Eq. (1). We inject a seed into the signal field and observe its output. This is somewhat similar to the method of stimulated emission tomography $[28,29]$. However, here, after the measurement of the output, we feed the result back to modify the input seed and iterate the process. This part is similar to the adaptive method of Polycarpou et al. [30]. To see what this leads to, we consider a coherent pulse of spectral shape $\alpha_{0}(\omega)$ injected into field $A$. Because of the orthonormality in Eq. (2), we can expand it as

$$
\alpha_{0}(\omega)=\sum_{k} \xi_{k} \psi_{k}(\omega)
$$

with $\xi_{k}=\int d \omega \psi_{k}^{*}(\omega) \alpha_{0}(\omega)$. Using Eq. (1) and assuming $\left|\xi_{k}\right|^{2} \gg 1$ to ignore spontaneous emission, we find

$$
\alpha^{\text {out }}(\omega)=\sum_{k} \xi_{k} \cosh G_{k} \psi_{k}(\omega)
$$

So, each mode is amplified but with different gain. Now let us exploit this gain difference: we can measure the output spectral function (amplitude and phase) and then program a new input field with a wave shaper according to the measured function. To keep the input power low, we can attenuate the output by a factor, say $\left(\cosh G_{1}\right)^{-1}$, so that the new input becomes

$$
\alpha_{1}(\omega)=\sum_{k} \xi_{k}\left(\cosh G_{k} / \cosh G_{1}\right) \psi_{k}(\omega) .
$$

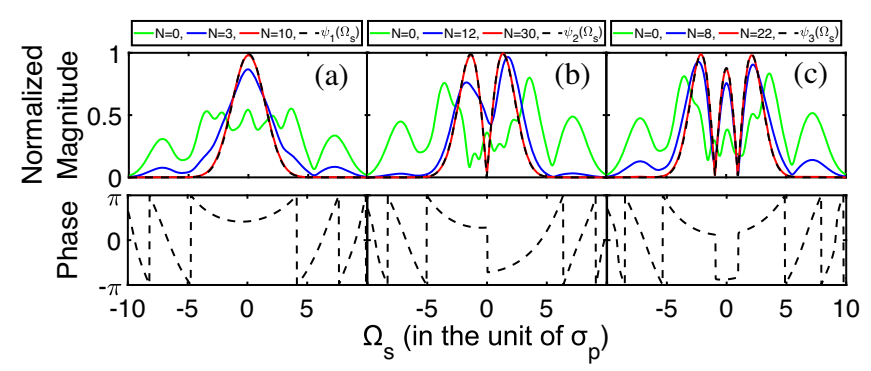

FIG. 2. Simulation results for the amplitudes (top) and converged phases (bottom) of the output spectral functions $\psi_{k}\left(\Omega_{s}\right)$ $\left(\Omega_{s} \equiv \omega_{s}-\omega_{s 0}\right.$ in the unit of pump bandwidth $\sigma_{p}$ ) for (a) $k=1$, (b) $k=2$, (c) $k=3$. The green curves are the input spectral functions, while the blue and red curves are intermediate outputs after the iteration steps indicated in the legends. The dashed curves are the final outputs. 


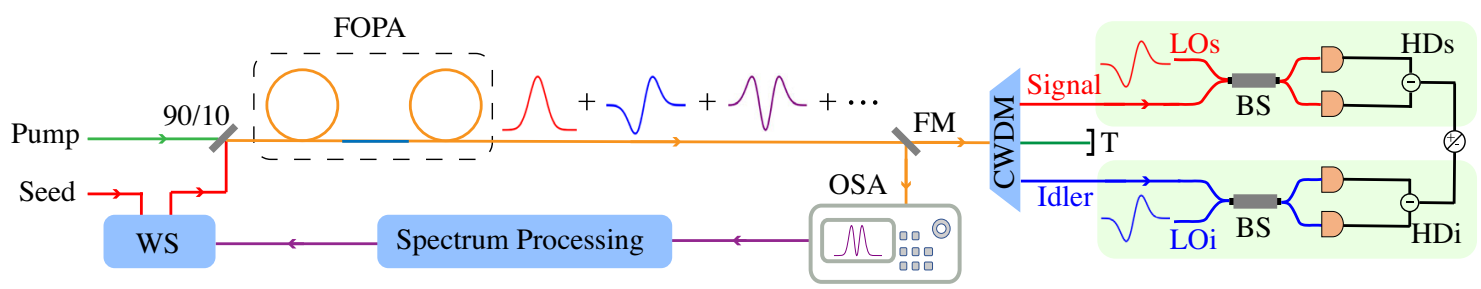

FIG. 3. Experimental setup. WS, wave shaper; FM, flip mirror; BS, beam splitter; $T$, terminator; HD, homodyne detection; FOPA, fiber optical parametric amplifier; OSA, optical spectrum analyzer; CWDM, coarse wavelength division multiplexer.

Since $G_{k}$ 's are different for different $k$, let us arrange mode order: $G_{1}>G_{2}>\cdots$ and $\cosh G_{k} / \cosh G_{1}<1$ for all except $k=1$. We iterate the procedure $N$ times and the field after $N$ iterations becomes

$$
\alpha_{N}(\omega)=\sum_{k} \xi_{k}\left(\cosh G_{k} / \cosh G_{1}\right)^{N} \psi_{k}(\omega)
$$

With $N$ large enough, $\left(\cosh G_{k} / \cosh G_{1}\right)^{N} \rightarrow 0$ for $k \neq 1$ and we are left with only the first mode: $\alpha_{N}(\omega) \propto \psi_{1}(\omega)$.

To obtain the mode function for $k=2$, we need to have an input field that is orthogonal to $\psi_{1}(\omega)$, that is, $\xi_{1}=0$. To achieve this, we use the Gram-Schmidt process: with $\psi_{1}(\omega)$ known, we set the input as $\alpha^{\prime}(\omega)=\alpha(\omega)-\xi_{1} \psi_{1}(\omega)$, which has $\xi_{1}^{\prime}=0$. Then the dominating mode is $k=2$. We perform orthogonalization after each iteration to ensure $\xi_{1}^{\prime}=0$. Subsequent modes can be obtained in a similar way but with the orthogonalization changed to $\alpha^{\prime}(\omega)=\alpha(\omega)-$ $\sum_{i=1}^{k-1} \xi_{i} \psi_{i}(\omega)$ for mode $k$.

In order to demonstrate the validity of the method, we run some simulations based on Eq. (8) for the JSF given in Ref. [5] but with a chirped pump phase of $e^{i \Omega^{2} / 2 \sigma_{p}^{2}}$ and set $G=2.5$. The results are shown in Fig. 2 for the first three modes. The green and dashed curves are the initial input and the final output spectral functions, respectively. The blue and red curves are for the intermediate steps with the step numbers shown in the legends. Because of overcrowdedness, only the final converged functions are shown for the phase part (bottom).

Experimental procedures and results.-The experimental setup is shown in Fig. 3, in which the pulse-pumped fiber optical parametric amplifier (FOPA) consists of two dispersion-shifted fibers and a single-mode fiber, which, through a quantum interference effect, modifies the JSF so that it is well behaved for the iteration method to converge [31] (see Supplemental Material [32] for details). The pump and the seed, with their path lengths carefully balanced through a delay line (not shown), are combined with a 90/10 beam splitter and simultaneously launched into the FOPA. The output of FOPA is either measured by an optical spectral analyzer (OSA) to determine the spectral profile or separated by coarse wavelength division multiplexer (CWDM) for quantum measurement by homodyne detection.
We first determine directly the temporal mode profiles of the fiber parametric amplifier by the feedback-iteration method described previously. For this, we use the recorded spectrum of the signal field by an OSA to reshape the input seed with a wave shaper (WS). Although an OSA only measures the spectral intensity, here, in the first order approximation, we assume that there is no dispersion in the phases of the mode functions, except a jump of $\pi$ at zeros for higher order modes. Such an assumption is valid because the spectral phases are relatively flat within the spectral width $(\sim 3.5 \mathrm{~nm})$ of the specially engineered source (see Ref. [31] and Supplemental Material [32]). So, we implement a sign change for the high order mode cases whenever the spectral intensity goes to zero. After a number of iterations ( $\sim 6-8$ depending on the shape of the initial injection), a steady shape is reached, which corresponds to one of the eigen-temporal modes from the parametric amplifier. We follow the steps described previously to find other eigen-temporal modes. The blue curves in Fig. 4 are the converged spectral intensity of the first three temporal modes [Figs. 4(a)-4(c)] together with those for the corresponding idler field [Figs. 4(d)-4(f)]. The curves are normalized to the maximum values. The dotted lines are the initially injected seed [only for Figs. 4(a) and 4(b)]. The pink curve in Fig. 4(a) is the

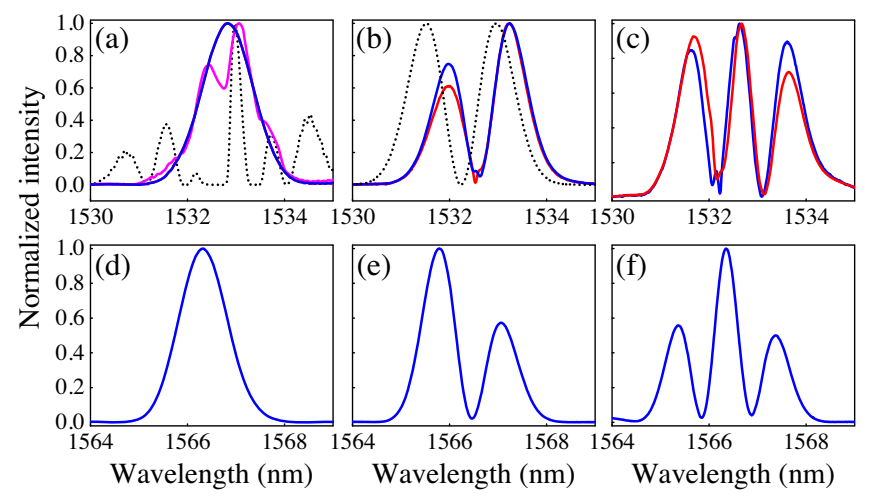

FIG. 4. Measured spectral intensity $\left|\psi_{k}(\omega)\right|^{2}$ for $k=$ (a) 1 , (b) 2, (c) 3, and those [(d)-(f)] for the corresponding idler field. The dotted lines are the initially injected seed [not shown for (c) due to crowdedness]. The pink curve in (a) is the output after two iterations. The blue lines are the output signal and the red lines are the feedback to the input [red is covered by blue in (a)]. 

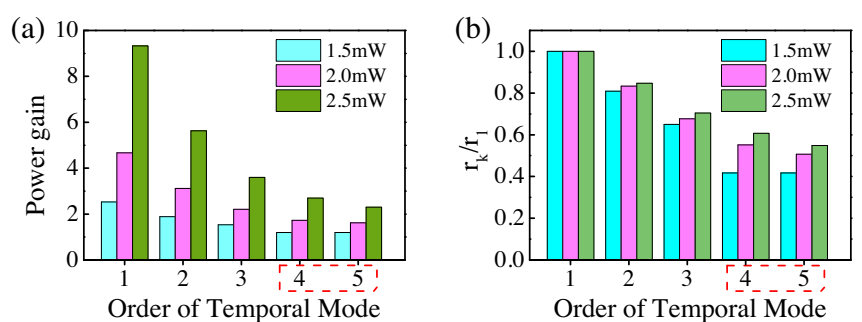

FIG. 5. (a) Power gain for the first five modes under different pump power. (b) Extracted mode numbers. The dashed red boxes on order numbers 4 and 5 indicate less reliable results due to unstable output.

output after only two iterations, showing fast convergence of the iteration. For the higher order modes $(k=2,3)$, there is a slight difference between the feedback input (red) and the output (blue). This is caused by the nonuniform spectral response of the detector, as well as dispersion in phase of the higher order modes.

The temporal mode structure is characterized by the distribution of the $G_{k}$ values, which can be obtained from the power gain $\cosh ^{2} G_{k}$ for each mode. The measured power gains for the first five modes under different pump powers are shown in Fig. 5(a) with the extracted $r_{k} / r_{1}\left(\equiv G_{k} / G_{1}\right)$ values shown in Fig. 5(b). The dashed red boxes on order numbers 4 and 5 in the figure indicate that the output is not stable. This is because the bandwidths of the higher orders are too broad and run outside the range of the well-behaved JSF and into the next region of the JSF (see Supplemental Material [32] on JSF). As can be seen from Fig. 5(b), the mode parameters $\left\{r_{k}\right\}$ change with pump power increase and the changes become more prominent for the high orders. Furthermore, we also observe some significant changes in the converged mode profiles (Fig. 4) as the pump power changes. This is in support of the theory in Refs. $[26,27]$ that mode structure changes with the pump power. However, the mode changes may also be caused by other nonlinear effects in fibers such as self-phase modulation, which occur at high pump power.

Once the temporal mode profiles are determined, we can perform homodyne detection with local oscillators (LOs and $\mathrm{LO} i$ ) tailored to match the specific temporal mode of our interest. Since temporal modes are orthogonal to each other, when the LO is matched to a specific mode, there is no contribution from other modes for the homodyne detection [5]. So we can use homodyne detection to select the mode of our interest. With this, we first check the correlation between different modes by measuring the covariance matrix $C_{m n} \equiv\left\langle\Delta \hat{O}_{m} \Delta \hat{O}_{n}\right\rangle / \sqrt{\left\langle\Delta^{2} \hat{O}_{m}\right\rangle\left\langle\Delta^{2} \hat{O}_{n}\right\rangle}$, where indices $m, n$ denote different modes and $\hat{O}$ is either the amplitude quadrature $\hat{X} \equiv \hat{a}+\hat{a}^{\dagger}$ or the phase quadrature $\hat{Y} \equiv\left(\hat{a}-\hat{a}^{\dagger}\right) / i$ when properly locking the phases between LOs (LOi) and signal (idler) beam to either 0 or $\pi / 2$ (see Supplemental Material [32] for the details). Figure 6 shows the results of either amplitude [Fig. 6(a)]
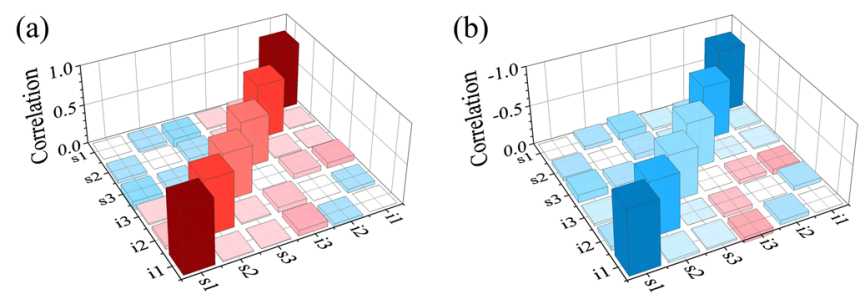

FIG. 6. Covariance matrix elements for (a) amplitude quadrature $(X)$ and (b) phase quadrature $(Y)$. The trivial diagonal elements of $C_{i i}=1$ are taken out, while the nonzero antidiagonal elements show strong pairwise correlation between the corresponding modes of signal and idler beams. Other near-zero, offdiagonal elements indicate total independence between different modes. See Supplemental Material [32] for exact numerical values.

or phase [Fig. 6(b)] quadrature for six modes of $\{s 1, s 2, s 3, i 3, i 2, i 1\}$ with $s k, i k(k=1,2,3)$ denoting the $k$ th order conjugate modes of signal and idler fields (for exact numerical values, see Supplemental Material [32]). We only measure up to 3 orders because higher orders are not stable (see Fig. 5). In Fig. 6, we take out the diagonal elements that are all equal to 1 . The antidiagonal elements correspond to $C_{s 1 i 1}, C_{s 2 i 2}, C_{s 3 i 3}$, whose nonzero values show the strong pairwise correlation (amplitude $\hat{X}$ ) or anticorrelation (phase $\hat{Y}$ ) between the corresponding signal and idler modes of the same order, whereas the other offdiagonal elements are near zero, indicating the total independence between different orders of the temporal modes and confirming the orthogonality of the modes.

Next, we check the quantum entanglement between the signal and idler fields by measuring $\left\langle\Delta^{2}\left(\hat{X}_{s k}-\hat{X}_{i k}\right)\right\rangle$ and $\left\langle\Delta^{2}\left(\hat{Y}_{s k}+\hat{Y}_{i k}\right)\right\rangle(k=1,2,3)$ for the $k$ th modes and verifying the inseparability criterion of entanglement: $I_{k} \equiv$ $\left\langle\Delta^{2}\left(\hat{X}_{s k}-\hat{X}_{i k}\right)\right\rangle /\left\langle\Delta^{2}\left(\hat{X}_{s k}-\hat{X}_{i k}\right)\right\rangle_{u}+\left\langle\Delta^{2}\left(\hat{Y}_{s k}+\hat{Y}_{i k}\right)\right\rangle /$ $\left\langle\Delta^{2}\left(\hat{Y}_{s k}+\hat{Y}_{i k}\right)\right\rangle_{u}<I_{u}=2$, with the subscript $u$ denoting the unentangled vacuum case [36]. At pump power of about $1.3 \mathrm{~mW}$, resulting in power gains of the FOPA of 2.1, 1.5 , and 1.3 for the first three modes, the measured values of $I_{k} / I_{u}(k=1,2,3)$ are obtained in log scale as -2.56 , -1.50 , and $-1.17 \mathrm{~dB}$ for the first three order modes, respectively.

Taking the total detection efficiency into consideration, we obtain the corrected value of $I / I_{u}$ as $-3.70,-2.00$, and $-1.60 \mathrm{~dB}$ or $I=0.85,1.26,1.38<2$ for the first three modes, respectively. This shows pairwise entanglement between the signal and idler modes of the same order. The worsening for the higher orders is mostly because of the decreasing gains for the higher order modes, but is also partly because of the mode mismatching due to inaccurate mode function measurement.

In summary, we show theoretically and demonstrate experimentally a method that directly determines the temporal or spectral profiles of the eigenmodes for the signal and idler fields generated from a fiber-based 
parametric amplifier pumped by a short pulse. We further show experimentally that they are pairwise entangled.

In our proof-of-principle experiment, we do not measure phase part of the pulses because we assume constant phase across the spectrum, which is fulfilled by the specially engineered source. Our experimental approach thus cannot measure any spectral phase variations and will not work for sources with large phase changes. Our simulations, on the other hand, demonstrate the general applicability of the method once intensity and phase are both considered (Fig. 2). So, for the general case, a full measurement in both intensity and phase is necessary. This can be done via pulse characterization techniques [37] such as frequencyresolved optical gating (FROG) [38], the interferometric method [39], and cross-correlation FROG [40].

We also demonstrate that the mode structure depends on the gain of the parametric amplifier. However, the method described here is only suitable for the high gain parametric amplifier, which gives rise to quantum entanglement in continuous variables. For the low gain case, which produces a two-photon entangled state, the method fails because the gain is near unity for all modes. Nevertheless, the feedbackiteration idea in the current method can still be applied to the low gain case by involving the stimulated emission in the idler field. The detail is presented in another paper [41].

The technique can be generalized to other pulse-pumped systems, such as frequency conversion processes or other degrees of freedom, such as spatial modes to find the eigenmodes of the system. So, the potential applications of the technique are not limited only to quantum optics but can be applied to classical systems as well.

We would like to thank S. Lemieux and R. W. Boyd for helpful discussion. This work was supported in part by National Natural Science Foundation of China (Grants No. 91836302, No. 91736105, and No. 11527808), the National Key Research and Development Program of China (Grant No. 2016YFA0301403), and in part by U.S. National Science Foundation (Grant No. 1806425).

${ }^{*}$ Corresponding authors.

xiaoyingli@tju.edu.cn

${ }^{\dagger}$ Corresponding authors.

zou@iupui.edu

[1] W. E. Lamb, Jr., Anti-photon, Appl. Phys. B 60, 77 (1995).

[2] B. Brecht, Dileep V. Reddy, C. Silberhorn, and M. G. Raymer, Photon Temporal Modes: A Complete Framework for Quantum Information Science, Phys. Rev. X 5, 041017 (2015).

[3] A. Christ, K. Laiho, A. Eckstein, K. N. Cassemiro, and C. Silberhorn, Probing multimode squeezing with correlation functions, New J. Phys. 13, 033027 (2011).

[4] W. Wasilewski, A. I. Lvovsky, K. Banaszek, and C. Radzewicz, Pulsed squeezed light: Simultaneous squeezing of multiple modes, Phys. Rev. A 73, 063819 (2006).
[5] Xueshi Guo, Nannan Liu, Xiaoying Li, and Z. Y. Ou, Complete temporal mode analysis in pulse-pumped fiberoptical parametric amplifier for continuous variable entanglement generation, Opt. Express 23, 29369 (2015).

[6] H. P. Yuen, Two-photon coherent states of the radiation field, Phys. Rev. A 13, 2226 (1976).

[7] D. C. Burnham and D. L. Weinberg, Observation of Simultaneity in Parametric Production of Optical Photon Pairs, Phys. Rev. Lett. 25, 84 (1970).

[8] R. E. Slusher, L. W. Hollberg, B. Yurke, J. C. Mertz, and J. F. Valley, Observation of Squeezed States Generated by Four-Wave Mixing in an Optical Cavity, Phys. Rev. Lett. 55, 2409 (1985).

[9] Z. Y. Ou and L. Mandel, Violation of Bell's Inequality and Classical Probability in a Two-Photon Correlation Experiment, Phys. Rev. Lett. 61, 50 (1988).

[10] Y. H. Shih and C. O. Alley, New Type of Einstein-PodolskyRosen-Bohm Experiment Using Pairs of Light Quanta Produced by Optical Parametric Down Conversion, Phys. Rev. Lett. 61, 2921 (1988).

[11] P. G. Kwiat, K. Mattle, H. Weinfurter, A. Zeilinger, A. V. Sergienko, and Y. Shih, New High-Intensity Source of Polarization-Entangled Photon Pairs, Phys. Rev. Lett. 75, 4337 (1995).

[12] Z. Y. Ou, S. F. Pereira, H. J. Kimble, and K. C. Peng, Realization of the Einstein-Podolsky-Rosen Paradox for Continuous Variables, Phys. Rev. Lett. 68, 3663 (1992).

[13] O. Aytür and P. Kumar, Pulsed Twin Beams of Light, Phys. Rev. Lett. 65, 1551 (1990).

[14] Y. Cai, J. Roslund, G. Ferrini, F. Arzani, X. Xu, C. Fabre, and N. Treps, Multimode entanglement in reconfigurable graph states using optical frequency combs, Nat. Commun. 8, 15645 (2017).

[15] C. Reimer, S. Sciara, P. Roztocki, M. Islam, L. Romero Cortés, Y. Zhang, B. Fischer, S. Loranger, R. Kashyap, A. Cino, S. T. Chu, B. E. Little, D. J. Moss, L. Caspani, W. J. Munro, J. Azana, M. Kues, and R. Morandotti, Highdimensional one-way quantum processing implemented on d-level cluster states, Nat. Phys. 15, 148 (2019).

[16] H. Wang, Y. He, Y.-H. Li, Z.-E. Su, B. Li, H.-L. Huang, X. Ding, M.-C. Chen, C. Liu, J. Qin, J.-P. Li, Y.-M. He, C. Schneider, M. Kamp, C.-Z. Peng, S. Höfling, C.-Y. Lu, and J.-W. Pan, High-efficiency multiphoton boson sampling, Nat. Photonics 11, 361 (2017).

[17] F. Hudelist, J. Kong, C. Liu, J. Jing, Z. Y. Ou, and W. Zhang, Quantum metrology with parametric amplifierbased photon correlation interferometers, Nat. Commun. 5, 3049 (2014).

[18] J. Roslund, R. M. de Araujo, S. Jiang, C. Fabre, and N. Treps, Wavelength-multiplexed quantum networks with ultrafast frequency combs, Nat. Photonics 8, 109 (2014).

[19] R. Schmeissner, J. Roslund, C. Fabre, and N. Treps, Spectral Noise Correlations of an Ultrafast Frequency Comb, Phys. Rev. Lett. 113, 263906 (2014).

[20] A. O. C. Davis, V. Thiel, and B. J. Smith, Measuring the quantum state of a photon pair entangled in frequency and time, arXiv:1809.03727.

[21] A. Eckstein, B. Brecht, and C. Silberhorn, A quantum pulse gate based on spectrally engineered sum frequency generation, Opt. Express 19, 13770 (2011). 
[22] B. Brecht, A. Eckstein, R. Ricken, V. Quiring, H. Suche, L. Sansoni, and C. Silberhorn, Demonstration of coherent time-frequency Schmidt mode selection using dispersionengineered frequency conversion, Phys. Rev. A 90, 030302 (R) (2014).

[23] D. V. Reddy, M. G. Raymer, and C. J. McKinstrie, Efficient sorting of quantum-optical wave packets by temporal-mode interferometry, Opt. Lett. 39, 2924 (2014).

[24] D. V. Reddy and M. G. Raymer, High-selectivity quantum pulse gating of photonic temporal modes using all-optical Ramsey interferometry, Optica 5, 423 (2018).

[25] Zhe-Yu Jeff Ou, Quantum Multi-Photon Interference (Springer, New York, 2007).

[26] N. Quesada and J.E. Sipe, Effects of time ordering in quantum nonlinear optics, Phys. Rev. A 90, 063840 (2014).

[27] P. R. Sharapova, G. Frascella, M. Riabinin, A. M. Pérez, O. V. Tikhonova, S. Lemieux, R. W. Boyd, G. Leuchs, and M. V. Chekhova, Properties of bright squeezed vacuum at increasing brightness, Phys. Rev. Research 2, 013371 (2020).

[28] M. Liscidini and J. E. Sipe, Stimulated Emission Tomography, Phys. Rev. Lett. 111, 193602 (2013).

[29] B. Fang, O. Cohen, M. Liscidini, J. E. Sipe, and V. O. Lorenz, Fast and highly resolved capture of the joint spectral density of photon pairs, Optica 1, 281 (2014).

[30] C. Polycarpou, K. N. Cassemiro, G. Venturi, A. Zavatta, and M. Bellini, Adaptive Detection of Arbitrarily Shaped Ultrashort Quantum Light States, Phys. Rev. Lett. 109, 053602 (2012).

[31] J. Su, L. Cui, J. Li, Y. Liu, Xiaoying Li, and Z. Y. Ou, Versatile and precise quantum state engineering by using nonlinear interferometers, Opt. Express 27, 20479 (2019).

[32] See Supplemental Material at http://link.aps.org/ supplemental/10.1103/PhysRevLett.124.213603 for experimental details, which includes Refs. [33-35].
[33] J. Su, L. Cui, J. Li, Y. Liu, X. Li, and Z. Y. Ou, Quantum state engineering by nonlinear quantum interference, arXiv: 1811.07646.

[34] R. Tang, J. Lasri, P. S. Devgan, V. Grigoryan, P. Kumar, and M. Vasilyev, Gain characteristics of a frequency nondegenerate phase-sensitive fiber-optic parametric amplifier with phase self-stabilized input, Opt. Express 13, 10483 (2005).

[35] Y, Liu, J. Li, L. Cui, N. Huo, S. M. Assad, Xiaoying Li, and Z. Y. Ou, Loss-tolerant quantum dense metrology with SU(1,1) interferometer, Opt. Express 26, 27705 (2018).

[36] L.-M. Duan, G. Giedke, J. I. Cirac, and P. Zoller, Inseparability Criterion for Continuous Variable Systems, Phys. Rev. Lett. 84, 2722 (2000).

[37] I. A. Walmsley and C. Dorrer, Characterization of ultrashort electromagnetic pulses, Adv. Opt. Photonics 1, 308 (2009).

[38] R. Trebino, K. W. DeLong, D. N. Fittinghoff, J. N. Sweetser, M. A. Krumbügel, B. A. Richman, and D. J. Kane, Measuring ultrashort laser pulses in the time-frequency domain using frequency-resolved optical gating, Rev. Sci. Instrum. 68, 3277 (1997).

[39] D. N. Fittinghoff, J. L. Bowie, J. N. Sweetser, R. T. Jennings, M. A. Krumbuegel, K. W. DeLong, R. Trebino, and I. A. Walmsley, Measurement of the intensity and phase of ultraweak, ultrashort laser pulses, Opt. Lett. 21, 884 (1996).

[40] J.-Y. Zhang, A. P. Shreenath, M. Kimmel, E. Zeek, R. Trebino, and S. Link, Measurement of the intensity and phase of attojoule femtosecond light pulses using OpticalParametric-Amplification Cross-Correlation FrequencyResolved Optical Gating, Opt. Express 11, 601 (2003).

[41] X. Chen, Xiaoying Li, and Z. Y. Ou, Direct temporal mode measurement of photon pairs by stimulated emission, Phys. Rev. A 101, 033838 (2020). 